\title{
¿Qué nos dicen las revistas y redes de investigación en trabajo social? Expresiones y concepciones en torno a la construcción de conocimiento disciplinar
}

\section{What do social work journals and research networks tell us? Expressions and conceptions around the construction of disciplinary knowledge}

\section{Núcleo Estudios Interdisciplinarios en Trabajo Social ${ }^{1}$}

Departamento de Trabajo Social, Universidad de Chile

Recibido: $29 / 10 / 2020$

Aceptado: 30/12/2020

\section{Cómo citar}

Núcleo Estudios Interdisciplinarios en Trabajo Social (2021). ¿Qué nos dicen las revistas y redes de investigación en trabajo social? Expresiones y concepciones en torno a la construcción de conocimiento disciplinar. Propuestas Críticas en Trabajo Social - Critical Proposals in Social Work 1(1),146-164. DOI: 10.5354/2735-6620.2021.61241

\section{Resumen}

En este artículo abordamos la cuestión de la construcción de conocimiento en trabajo social analizando las revistas académicas y las redes de investigación en tanto dimensiones clave en la diseminación de la producción intelectual de la disciplina. Se identifican singularidades y se problematizan críticamente los enfoques conceptuales, así como los estándares metodológicos y éticos. Se analiza la

Palabras clave: producción de conocimiento; investigación; revistas; redes; trabajo social.

El estudio fue realizado por Gianinna Muñoz-Arce, Gabriela Rubilar-Donoso, Teresa Matus-Sepúlveda y Paula Parada-Ballesteros, integrantes del Núcleo Estudios Interdisciplinarios en Trabajo Social, NEITS. Más información en www.neits.cl. 
configuración de los equipos, las líneas temáticas, la locación geográfica y el alcance político de los debates impulsados en las revistas indexadas en los catálogos más exigentes y en las redes de investigación en Europa, Estados Unidos, Brasil y Chile. Se plantea que la forma en que se produce conocimiento en trabajo social es "insular" o geopolíticamente concentrada, con asimetrías observables en términos de fundamentación conceptual y metodológica, diversa en configuración de los equipos y con alcances de incidencia pública también diferenciados. Se observa que las revistas tienden a reproducir lógicas endogenistas; mientras que las redes de investigación que funcionan con lógicas descentradas o heterárquicas están orientadas a abrir espacios de relaciones y a ejercer influencia en el debate público, así como a manifiestas preocupaciones en torno a la formación de futuros investigadoras/es desde la disciplina. Estos hallazgos permiten problematizar las lógicas de las políticas de fomento de la investigación y difusión del conocimiento -fondos públicos, incentivos, políticas de acceso- y proyectar los desafíos de trabajo social en términos de su posicionamiento y contribuciones al debate en esta materia.

\section{Abstract}

In this article we address the question of the construction of knowledge in social work by analyzing academic journals and research networks as key dimensions in the dissemination of the intellectual production of the discipline. Singularities are identified and conceptual approaches, as well as methodological and ethical standards, are critically problematized. The configuration of the teams, the thematic lines, the geographical location and the political scope of the debates promoted in journals and research networks are examined. It is proposed that the way in which knowledge is produced in social work is "insular" or geopolitically concentrated, with observable asymmetries on a conceptual and methodological basis, diversity in the configuration of teams and with different public impact scopes. It is observed that journals tend to reproduce endogenic logics; while the research networks that work with decentralized or heterarchical logics are oriented to open spaces for relationships and to exert influence in the public debate, as well as to manifest concerns about the training of future researchers from the discipline. These findings make it possible to problematize the logic of the policies to promote research and dissemination of knowledge -public funds, incentives, access policies- and to project the challenges of social work in terms of its positioning and contributions to the debate on this matter.

\section{Introducción}

La producción de conocimiento en trabajo social se ha expandido significativamente en las últimas décadas, siendo alentador el aumento de revistas académicas y el desarrollo de redes de investigación impulsadas en este período. Sin duda su creación ha aportado de manera importante al debate disciplinar y a la consolidación del conocimiento producido por generaciones de trabajadoras/es sociales que han abierto campos de estudio, espacios de difusión del conocimiento e intercambio de las producciones,

Keywords: knowledge production; research; journals; networks; social work. 
debates y reflexiones a nivel nacional e internacional (Taylor y Sharland, 2015).

Ahora bien, reconociendo esta expansión de la discusión disciplinar, surge el cuestionamiento respecto de las características y alcances que dicha discusión adopta en el contexto contemporáneo, asumiendo que detrás de cada revista académica o red de investigación que se crea hay apuestas y propuestas que son singulares. Las políticas nacionales de fomento a la investigación y los circuitos de producción científica a escala global influencian y dan forma a los alcances, políticas editoriales, ámbitos de estudio y objetivos de trabajo para revistas académicas y redes de investigación. En este sentido, la impronta del capitalismo cognitivo, la globalización y las demandas por mayores impactos del conocimiento generado (Zuchowski et al. 2019) han marcado la agenda del trabajo social en los últimos años.

En este marco, hemos desarrollado un estudio que analiza las características de las revistas académicas indexadas en los catálogos más exigentes y que gozan de mayor legitimidad desde el punto de vista de las políticas de investigación en Chile, y de las redes de investigación desarrolladas en Europa, Estados Unidos, Brasil y Chile. El propósito del estudio ha sido explorar la singularidad de revistas y redes e identificar los enfoques conceptuales predominantes, la configuración de las propuestas en términos disciplinares, metodológicos y geopolíticos, y sus alcances en términos de discusión pública, para, a partir de los hallazgos, problematizar y proyectar los desafíos de la discusión disciplinar en materia de producción de conocimientos en trabajo social de cara a sus cien años de existencia en Chile y América Latina

\section{La producción de conocimientos en la era del capitalismo cognitivo}

Los impactos del capitalismo en la producción de conocimiento han sido ampliamente estudiados en las últimas décadas (Blondeau et al., 2004; Vercellone, 2013; Ossa, 2016; Murray y Peetz, 2020). La instalación de lógicas de mercado en la investigación y las publicaciones - que se traducen en indicadores de productividad investigativa o bonos de incentivo a la publicación, por ejemplo- han vaciado de sentido ideas como "impacto" o "innovación" (Bourdieu, 1998), fomentando el trabajo individual en detrimento de la colaboración, y reforzando la competencia al interior de los equipos de trabajo. Es la era del capitalismo cognitivo, una nueva fase de acumulación que implica una nueva geopolítica donde el conocimiento ocupa un lugar central, poniendo de relieve los flujos de poder que circulan en el mundo global, donde "la propiedad intelectual, la concentración del conocimiento y las formas de reproducción social modelan la producción del conocimiento socialmente útil” (D’Amico, 2016, p.432). La producción de conocimientos en la era del capitalismo cognitivo está marcada por una 
orientación hacia el crecimiento y competitividad de las naciones, la cual se basa en los incentivos para acrecentar el capital intelectual, es decir, los y las trabajadoras del conocimiento - knowledge workers - y los servicios informáticos, enseñanza y formación (Blondeau et al., 2004).

Estas orientaciones han influenciado directamente las políticas de fomento de la investigación en Chile, así como en el resto del mundo. Fardella et al. (2017; 2019) han mostrado en sus estudios cómo la producción de conocimientos está organizada y controlada por dispositivos de gestión que permiten cuantificar y monitorear el trabajo académico, lo que da cuenta de los impactos del enfoque de la nueva gestión pública en la generación de conocimiento. De ahí que las políticas de fomento a la investigación se orienten al incentivo de todo aquello que pueda ser "vendido" o "transferido" a estudiantes, empresas y gobiernos. Es el caso de las patentes, publicación en revistas científicas pagadas, entre otras formas de mercantilización del conocimiento (Torres, 2014).

En el caso de trabajo social, investigaciones previas han permitido identificar que la inserción de trabajadoras/es sociales en esta nueva economía del conocimiento ha seguido un patrón relativamente similar al de otras disciplinas de las ciencias sociales (Figueroa et al., 2018; Muñoz y Rubilar, 2020).

El Trabajo Social nació en Chile hace ya casi un siglo con una vocación de cientificidad. Así queda en evidencia en estudios históricos que indagan en las primeras décadas del desarrollo de la disciplina. Matus, Aylwin y Forttes afirman que el trabajo social emerge con una dirección científica, y citando a Elena Hott señalan que "le interesa perfeccionar sus métodos e investigar científicamente sus problemas" (Hott, 1930, p.350 en Matus et al., 2004, p. 47). Esta orientación también está presente en la gestación de la disciplina en otros países como Estados Unidos e Inglaterra articulando las nociones de ciencia, conocimiento y transformación (Deegan, 1997; Miranda, 2003; Travi, 2011; Álvarez-Uría y Parra, 2014; Reininger, 2018). En este sentido, el trabajo social tiene en sus orígenes un proyecto global de ciencia sociales con vocación de cientificidad (Morera, 2010), lo que redunda en su identidad disciplinaria, que entre otras funciones debe mediar entre la producción de conocimiento social y sus aplicaciones.

Sin embargo, el desarrollo y difusión de la discusión disciplinar en Chile es menos visible si se compara con la situación del trabajo social en otros países. Existe alrededor de una docena de revistas de trabajo social en el país -solo 3 de ellas indexadas en el catálogo ERIH Plus- y una Red de Investigadoras/es en Trabajo Social creada hace 5 años. Debido a que las políticas de fomento a la investigación premian las 
publicaciones en revistas indexadas en los catálogos Web of Science (WoS) y Scopus -y a que ninguna publicación de trabajo social chilena está indexada en estos catálogos- la mayoría de la publicación "competitiva” apunta a dichas revistas (Muñoz, 2018; Muñoz y Rubilar, 2020). Las redes de investigación, como contrapartida, apuestan por expandir vínculos y crear comunidades de sentido e intercambio de conocimientos. Revistas y redes, de alguna manera, operan como dos aristas de la producción de conocimientos en trabajo social en el contexto actual, que, a su vez, obedecen a lógicas de justificación diferenciadas, incluso opuestas. Bajo las interrogantes sobre el tipo de publicación que hoy día prevalece en el trabajo social -debido a su legitimación por las políticas de fomento a la investigación- y el tipo de redes de investigación que se han desarrollado en los últimos años, se llevó a cabo el estudio que se presenta a continuación.

\section{Metodología}

Se analizaron todas las revistas de trabajo social indexadas en WoS, Scopus, Erih Plus y Scielo vigentes en las bases de datos oficiales durante el mes de julio 2020. En total, se incluyeron en este análisis 30 revistas de trabajo social indexadas WoS, 18 en Scopus, 15 en Erih Plus y 4 en Scielo (Ver Cuadro $N^{\circ} 1$ ). A partir de una exhaustiva revisión de sus sitios web, se analizó el marco de definiciones generales de cada revista y también los contenidos del último número publicado en cada una de ellas. Se consideraron estas revistas debido a su alto reconocimiento por parte de las políticas de fomento a la investigación a escala nacional e internacional.

En lo que respecta a las redes de investigación en trabajo social, se analizaron cuatro iniciativas: Society for Social Work and Research (SSWR) de Estados Unidos, European Social Work Research Association (ESWRA), la Associação Brasileira de Ensino e Pesquisa em Serviço Social (ABEPSS) y la Red de Investigadores en Trabajo Social de Chile, estudiando las declaraciones y materiales expuestos en sus sitios web durante el mes de agosto de 2020 (Ver Cuadro $\mathrm{N}^{\circ}$ 2).

A partir de las revisiones realizadas, se organizaron matrices de análisis temático identificando la información relevante para cada una de las 7 dimensiones establecidas: 1) enfoques conceptuales, 2) estándares metodológicos, 3) estándares éticos, 4) configuración de los equipos, 5) líneas temáticas, 6) locación geográfica y 7) alcance político de los debates. Los datos fueron discutidos en reuniones de análisis que dieron lugar a la síntesis que se presenta a continuación. 


\section{Cuadro $\mathrm{N}^{\circ}$ 1: Revistas académicas de trabajo social analizadas}

\begin{tabular}{|c|c|c|}
\hline \multicolumn{2}{|r|}{ Revista } & Sitio web \\
\hline & & $\begin{array}{l}\text { Web of Science (WoS) } \\
\text { Fuente: https://mjl.clarivate.com/home }\end{array}$ \\
\hline 1 & Affilia & https://journals.sagepub.com/home/aff \\
\hline 2 & Asian Social Work and Policy Review & https://onlinelibrary.wiley.com/journal/17531411 \\
\hline 3 & Australian Social Work & https://www.tandfonline.com/toc/rasw20/current \\
\hline 4 & Child and Family Social Work & https://onlinelibrary.wiley.com/journal/13652206 \\
\hline 5 & Child Development & https://srcd.onlinelibrary.wiley.com/journal/14678624 \\
\hline 6 & Clinical Social Work Journal & https://www.springer.com/journal/10615 \\
\hline 7 & Health and Social Work & https://academic.oup.com/hsw \\
\hline 8 & Human Service Organizations: & \\
\hline & Management, Leadership \& Governance & https://www.tandfonline.com/toc/wasw21/current \\
\hline 9 & International Journal of Social Welfare & https://onlinelibrary.wiley.com/journal/14682397 \\
\hline 10 & International Social Work Journal & https://journals.sagepub.com/home/isw \\
\hline 11 & Journal of Gerontological Social Work & https://www.tandfonline.com/toc/wger20/current \\
\hline 12 & Journal of The Society for Social & \\
\hline & Work and Research & https://www.journals.uchicago.edu/toc/jsswr/current \\
\hline 13 & Journal of Social Work & https://journals.sagepub.com/home/jsw \\
\hline 14 & Journal of Social Work Education & https://www.tandfonline.com/toc/uswe20/current \\
\hline 15 & Journal of Social Work Practice & https://www.tandfonline.com/toc/cjsw20/current \\
\hline 16 & Qualitative Social Work & https://journals.sagepub.com/home/qswa \\
\hline 17 & Research on Social Work Practice & https://journals.sagepub.com/home/rsw \\
\hline 18 & Smith College Studies in Social Work & https://www.tandfonline.com/toc/wscs20/current \\
\hline 19 & Social Work & https://academic.oup.com/sw \\
\hline 20 & Social Work in Health Care & https://www.tandfonline.com/toc/wshc20/current \\
\hline 21 & Social Work in Public Health & https://www.tandfonline.com/toc/whsp20/current \\
\hline 22 & Social Work Research & https://academic.oup.com/swr \\
\hline 23 & The Indian Journal of Social Work & https://www.tiss.edu/view/6/research/the-indian-journal-of-social-work/ \\
\hline 24 & Journal of Social Policy & https://www.cambridge.org/core/journals/journal-of-social-policy/latest-issue \\
\hline 25 & Journal of Social Service Research & https://www.tandfonline.com/toc/wssr20/current \\
\hline 26 & Social Policy and Administration & https://onlinelibrary.wiley.com/journal/14679515 \\
\hline 27 & Social Service Review & https://www.journals.uchicago.edu/toc/ssr/current \\
\hline 28 & Social Work \& Social Sciences Review & https://journals.whitingbirch.net/index.php/SWSSR \\
\hline 29 & Journal of Comparative Social Work & https://journals.uis.no/index.php/JCSW \\
\hline 30 & The British Journal of Social Work & https://academic.oup.com/bjswhttps://www.tandfonline.com/toc/wswe20/current \\
\hline
\end{tabular}




\begin{tabular}{|c|c|c|}
\hline \multicolumn{2}{|r|}{ Revista } & Sitio web \\
\hline \multicolumn{3}{|c|}{$\begin{array}{c}\text { SCOPUS } \\
\text { Fuente: https://wwwscopus.com/source }\end{array}$} \\
\hline 1 & African Journal of Social Work & https://www.ajol.info/index.php/ajsw \\
\hline 2 & Annual of Social Work & https://hrcak.srce.hr/ljetopis \\
\hline 3 & Asian Social Work and Policy Review & https://onlinelibrary.wiley.com/journal/17531411 \\
\hline 4 & China Journal of Social Work & https://www.tandfonline.com/loi/rcsw20 \\
\hline 5 & Critical and Radical Social Work & https://www.ingentaconnect.com/content/tpp/crsw \\
\hline 6 & Child and Adolescent Social Work Journal & https://www.springer.com/journal/10560 \\
\hline 7 & Journal of Analytic Social Work & https://www.tandfonline.com/toc/wzsw20/current \\
\hline 8 & Journal of Ethnic and Cultural Diversity in Social Work & https://www.tandfonline.com/toc/wecd20/current \\
\hline 9 & Journal of Evidence-Based Social Work & https://www.tandfonline.com/toc/webs20/current \\
\hline 10 & Journal of Family Social Work & https://www.tandfonline.com/toc/wfsw20/current \\
\hline 11 & Journal of Religion and Spirituality in Social Work & https://www.tandfonline.com/toc/wrsp20/current \\
\hline 12 & Journal of Social Work in End-Of-Life and Palliative Care & https://www.tandfonline.com/toc/wswe20/current \\
\hline 13 & Journal of Social Work Practice in The Addictions & https://www.tandfonline.com/toc/wswp20/current \\
\hline 14 & Journal of Teaching in Social Work & https://www.tandfonline.com/toc/wtsw20/current \\
\hline 15 & Social Work Education & https://www.tandfonline.com/toc/cswe20/current \\
\hline 16 & Social Work in Mental Health & https://www.tandfonline.com/toc/wsmh20/current \\
\hline 17 & Social Work/Maatskaplike Werk & https://socialwork.journals.ac.za/pub \\
\hline 18 & Social Work with Groups & https://www.tandfonline.com/toc/wswg20/current \\
\hline \multicolumn{3}{|c|}{$\begin{array}{c}\text { ERIH PLUS } \\
\text { Fuente: https://dbh.nsd.uib.no/publiseringskanaler/erihplus/ }\end{array}$} \\
\hline 1 & Alternativas. Cuadernos de trabajo social. & https://alternativasts.ua.es/ \\
\hline 2 & $\begin{array}{l}\text { Anuarul Universitatii "Petre Andrei"din Iasi - Fascicula: } \\
\text { Asistenta Sociala, Sociologie, Psihologie }\end{array}$ & https://lumenpublishing.com/journals/index.php/upa-law \\
\hline 3 & Clinical Social Work and Health Intervention & https://clinicalsocialwork.eu/ \\
\hline 4 & Conciencia Social - Revista Digital de Trabajo Social & https://revistas.unc.edu.ar/index.php/ConCienciaSocial \\
\hline 5 & Cuadernos de Trabajo Social (Chile) & https://cuadernots.utem.cl/ \\
\hline 6 & Cuadernos de Trabajo Social (España) & https://revistas.ucm.es/index.php/CUTS \\
\hline 7 & Czech and Slovak Social Work & http://www.socialniprace.cz/eng/index.php \\
\hline 8 & $\begin{array}{l}\text { Global Social Work: journal of social intervention } \\
\text { research }\end{array}$ & https://revistaseug.ugr.es/index.php/tsg \\
\hline 9 & $\begin{array}{l}\text { Ehquidad. International Welfare Policies and Social } \\
\text { Work Journal }\end{array}$ & https://revistas.proeditio.com/ehquidad/ \\
\hline 10 & European Journal of Social Work & https://www.tandfonline.com/toc/cesw20/current \\
\hline 11 & $\begin{array}{l}\text { Scientific Annuals of Alexandru Ioan Cuza } \\
\text { University of Iasi. Sociology and Social Work }\end{array}$ & https://anale.fssp.uaic.ro/index.php/asas \\
\hline 12 & Social Work Review & http://www.swreview.ro/index.pl/home_en \\
\hline 13 & Social Work \& Society & http://ejournals.bib.uni-wuppertal.de/index.php/sws/ \\
\hline 14 & Trabajo Social Hoy & http://www.trabajosocialhoy.com/ \\
\hline 15 & Voces desde El Trabajo Social & https://revistavocests.org/index.php/voces \\
\hline \multicolumn{3}{|c|}{$\begin{array}{c}\text { SCIELO } \\
\text { Fuente: https://scielo.org/en/journals/list-by-subject-areal }\end{array}$} \\
\hline 1 & $\begin{array}{l}\text { Prospectiva. Revista de Trabajo Social e Intervención } \\
\text { Social }\end{array}$ & https://revistaprospectiva.univalle.edu.co/ \\
\hline 2 & Servicio Social Y Sociedades & $\begin{array}{l}\text { https://www.scielo.br/scielo.php?script=sci_erial\&pi } \\
\mathrm{d}=0101-6628 \& \operatorname{lng}=\text { =es\&nrm=iso }\end{array}$ \\
\hline 3 & Trabajo Social & $\begin{array}{l}\text { https://revistas.unal.edu.co/index.php/tsocial/issue/ } \\
\text { view/5171 }\end{array}$ \\
\hline 4 & Katálysis & $\begin{array}{l}\text { https://www.scielo.br/scielo.php?script=sci_serial\&pi } \\
\mathrm{d}=1414-4980 \& \operatorname{lng}=\text { es\&nrm=iso }\end{array}$ \\
\hline
\end{tabular}

Fuente: elaboración propia. 


\section{Las revistas académicas en trabajo social}

Al analizar el corpus de revistas de trabajo social indexadas en WoS, Scopus, Erih Plus y Scielo, se observan dos tendencias, que hemos codificado como revistas tipo A y revistas tipo $\mathrm{B}$.

Revistas tipo A: Las revistas indexadas en WoS y Scopus, en su gran mayoría, no explicitan un enfoque conceptual en específico. Aceptan artículos escritos desde diversos enfoques o que derechamente no explicitan una perspectiva conceptual en particular. Sin embargo, el requerimiento en términos de estándares metodológicos y éticos es alto, posiblemente porque la propia indexación WoS y Scopus lo exige como requisito para todas sus revistas. La gran mayoría de los artículos que se publican en estas revistas proviene de autores/as del mundo angloamericano, y con estudios de trabajo social a nivel de postgrado. La gran mayoría de los artículos corresponden a reportes de investigación empírica. Las revistas en general no tienen líneas de investigación definidas, aunque dominan temáticas referidas a la intervención clínica e intervención con familias, y análisis de la posición de los/as trabajadores sociales en los procesos de intervención social. Destacan en esta última línea los estudios sobre burn-out, cansancio crónico y estrés profesional. Son escasos los artículos que reportan análisis estructurales de los problemas de intervención del trabajo social, y la mayoría de los artículos trabaja las temáticas o fenómenos sociales poniendo el acento en los individuos que sufren los problemas, no en los mecanismos estructurales que los producen.

Revistas tipo B: Las revistas indexadas en Erih Plus y Scielo, por su parte, muestran un perfil diametralmente opuesto en la mayoría de las dimensiones estudiadas. Las revistas indexadas en Erih Plus son generalmente españolas, mientras que, de las indexadas en Scielo, dos son brasileñas y dos son colombianas. En las revistas Erih Plus el alcance de las publicaciones es más cosmopolita, aunque se observa la tendencia a centrarse en el análisis de la realidad española; igualmente sucede con las revistas indexadas en Scielo, las cuales ponen el foco en la discusión del trabajo social en América Latina. Los alcances políticos del análisis de los fenómenos sociales son mucho más claros en este grupo de revistas, especialmente en aquellas indexadas en Scielo. A diferencia de las revistas WoS y Scopus, que ponen acento en el estudio de la figura del/la trabajadora social en sí mismo, en las revistas Erih Plus, y especialmente Scielo, no se observa tal tendencia. Más bien el énfasis de las temáticas publicadas dice relación con la violencia y las diversas manifestaciones de la exclusión que se vuelven problemas urgentes de resolver por el trabajo social: la pobreza, el racismo, los feminicidos, entre muchos otros. Los antecedentes que permiten evaluar el nivel de exigencia de los estándares éticos y metodológicos que deben cumplir los artículos para 
ser publicados son heterogéneos (algunas revistas entregan detalles de ello, otras no). Estos hallazgos sugieren que existen dos realidades paralelas en lo que refiere a la producción del conocimiento disciplinar que se plasma en las revistas: revistas A que publican conocimiento que proviene de la investigación empírica, con un fuerte foco en metodología (cualitativa, cuantitativa y mixta) y con estándares éticos muy elevados; pero con una lectura de los problemas sociales centrada en el individuo, con escasa discusión conceptual y débil análisis de las implicancias políticas de los resultados de las investigaciones reportadas, donde el/la trabajadora social ocupa un lugar central como objeto de estudio. Y, por otra parte, las revistas tipo B, que publican menos artículos empíricos centrándose especialmente artículos en formato de ensayo, que discuten conceptual y políticamente los hallazgos o propuestas, que se centran en problemáticas urgentes, quedando fuera del foco de interés la propia figura del trabajador social. Todas (revistas A y B) tienen un énfasis geográfico, es decir, el trabajo social producido en cada región se auto observa, limitando el alcance cosmopolita de la producción de conocimientos y más aún el intercambio Sur-Norte. Muy consistente con este hallazgo, Roche y Flynn (2018) señalan que las publicaciones en las revistas de trabajo social de indexación más valorada por la comunidad académica -como WoS y Scopus- reproducen una lógica colonial de producción de conocimiento que se manifiesta en que menos de un $10 \%$ de los artículos publicados en estas revistas han sido escritos por autoras/es del Sur Global, e identifican importantes brechas de conocimiento en términos de teoría e investigación disciplinar. Se trata, claramente, de otra expresión del capitalismo cognitivo con sesgo colonial (Lee y Lee, 2013).

A ello se suma la problemática por el tipo de acceso que tienen estas publicaciones: las revistas tipo A, WoS y la mayoría de las indexadas en Scopus, no cuentan con acceso abierto, a diferencia de las revistas Erih Plus y Scielo que suelen tenerlo. El pago que debe realizarse para acceder a estas publicaciones oscila entre los USD 40,00 (por un acceso de 24 horas a un artículo específico) hasta los USD 345,00 (por un acceso de un mes a un número de una revista). Esta situación, además de reproducir el carácter elitista de la producción de conocimientos en trabajo social, refuerza la reproducción geopolítica del conocimiento válido -que es construido por quienes pueden acceder a él e interpelarlo desde sus mismos códigos (Alperín y Fischman, 2015; Tarragó et al., 2018).

Esto tiene implicancias cruciales para la discusión disciplinar y para la forma de generación de conocimiento que es legitimada hoy en día por las políticas de fomento de la investigación (Sierra y Alberich, 2019): estos dos mundos paralelos -las revistas tipo A y tipo B (sin considerar siquiera toda la amplia gama de revistas que tienen una indexación de menor relevancia o que no están indexadas) obedecen a una lógica que 
reproduce una geopolítica del conocimiento (Lander, 1999) y la validación de trabajos que no plantean una orientación conceptual y política explícita -casi al borde de una pretensión de neutralidad que gana legitimidad en tanto justifica sus procedimientos metodológicos con rigurosidad (Smith, 2006; Lee y Lee, 2013). Ciertamente, es necesario remarcar que hay excepciones a esta tendencia. Es el caso de revistas tipo A que no solo aceptan, sino que valoran la discusión conceptual y el debate de los alcances políticos de los resultados de investigación a una escala de cuestionamiento de los sistemas, estructuras y mecanismos contextuales que producen la opresión. Es el caso de la Revista Affilia, basada en perspectivas feministas, o la revista Critical and Radical Social Work, como su nombre lo indica, está fundada en diversas vertientes del pensamiento crítico y radical. También existen revistas que valoran estas discusiones conceptuales y políticas pero que no la intencionan explícitamente, como por ejemplo la revista International Social Work (Durham University) o el British Journal of Social Work (Asociación Británica de Trabajadoras/es Sociales).

La reproducción de esta geopolítica del conocimiento y de una aparente neutralidad en la argumentación expuesta en muchos de los artículos publicados en las revistas tipo A, encierra una paradoja: tiene más valor publicar en revistas tipo A (del norte) para ganar fondos de investigación en nuestros países (del sur). Decimos que es una paradoja porque además estas revistas tipo A no garantizan acceso abierto (Serrano y Prats, 2005; Lillis y Curry, 2010), teniendo que destinarse fondos públicos (de investigaciones concursables, por ejemplo) para costear dicho acceso por parte de los autores/as -cabe considerar que para que un artículo tenga acceso abierto, su autor/a puede tener que llegar a costear los USD 3,900, algo así como tres millones de pesos chilenos. Resulta problemático que se deba pagar con fondos públicos, el acceso que "venden" entidades privadas como las editoriales que son propietarias de las revistas de trabajo social más prestigiosas a nivel mundial (Taylor \& Francis, Routledge, Sage, Wiley-Blackwell, entre otras). En Europa se está regulando esta cuestión: si los resultados fueron producidos por una investigación con fondos públicos, los artículos solo deben publicarse en revistas de acceso abierto ${ }^{2}$. El debate en Chile se ha iniciado en la última década, pero aún falta bastante camino por recorrer en esta discusión.

\section{Las redes de investigación en trabajo social}

Al analizar las cuatro redes de investigación - Society for Social Work and Research de Estados Unidos (SSWR), European Social Work Research Association (ESWRA), la Associação Brasileira de Ensino e Pesquisa em Serviço Social (ABEPSS) y la Red de Investigadores en Trabajo Social de Chile- se observaron diferencias en términos de la

2 Para mayor detalle de esta discusión, ver https://www.scienceeurope.org/coalition-s/ 
consolidación de estas iniciativas. Por ejemplo, la SSWR y la ABEPSS tienen una vigencia de más de veinticinco años y por lo mismo se encuentran consolidadas en sus respectivos países. Entre sus líneas de acción incluyen actividades de formación para pre y postgrado y lideran una revista que es editada desde la asociación: Journal of de Society for Social Work and Research (JSSWR) y Revista Temporalis, respectivamente. Por su parte, la ESWRA y la Red de Investigadores en Trabajo Social de Chile son asociaciones creadas en los últimos cinco años, por lo mismo su desarrollo y alcance aún no se ha consolidado.

\section{Cuadro $\mathrm{N}^{\circ}$ 2: Redes de investigación en trabajo social analizadas}

\begin{tabular}{|c|l|l|l|}
\hline & Nombre de la red & país & sitio web \\
\hline 1 & Society for Social Work and Research - SSWR & Estados Unidos & https://secure.sswr.org/ \\
\hline 2 & European Social Work Research Association -ESWRA & Europa & https://www.eswra.org/ \\
\hline 3 & ABEPPS - Brasil Grupos Temáticos de Pesquisa & Brasil & http://www.abepss.org.br/ \\
\hline 4 & Red de Investigadores en Trabajo Social de Chile & Chile & https://redinvestigaciontrabajosocial.cl/ \\
\hline
\end{tabular}

Fuente: elaboración propia.

Respecto de las siete dimensiones de análisis, se observa que sólo la SSWR declara una perspectiva conceptual desde donde se posicionan sus investigaciones y acciones como red, sustentadas en el enfoque basado en evidencia. En las otras tres redes no se explicita un posicionamiento en este sentido, aunque es posible inferir de sus líneas de investigación que ABEPSS lo hace desde un enfoque de trabajo social crítico. Los estándares metodológicos son altos y explícitos para SSWR y de menor exigencia para la Red de Investigadores en Trabajo Social de Chile y la ESWRA, mientras que en ABEPSS no hay una definición de este tipo de estándares. Las exigencias éticas son explícitas en las dos redes de mayor trayectoria en el tiempo (SSWR y ABEPSS), mientras que en las redes más recientes este aspecto no es abordado explícitamente.

Las estructuras y modalidades de funcio namiento de la red también difieren. En el caso de las redes más consolidadas se observa un directorio y normas explicitas sobre su funcionamiento, lo que sigue una tendencia similar en la red europea. En estos tres casos es posible observar una gobernanza institucionalizada que supone además la administración de recursos. En ese sentido las redes con esta estructura más formalizada incluyen algunas modalidades o estrategias de financiamiento a la investigación. A diferencia de las otras asociaciones, la Red de Investigadores en Trabajo Social de Chile es más bien un colectivo disciplinar de personas que 
desarrollan funciones en torno a un objetivo común, su conformación es más horizontal y no posee una estructura jerárquica reconocible.

Las redes suponen articulación de temas e intereses. En ese sentido en SSWR se identifican seis ejes temáticos centrales: Investigación, capacitación e innovación en prácticas de trabajo social; Programas de intervención y políticas públicas y políticas sociales, programas y sistemas de salud. En ABEPPS las líneas de investigación declaradas son: Desarrollo, práctica y utilización de la investigación del trabajo social, Debates meta disciplinarios y enfoques teóricos en trabajo social, Educación y formación en trabajo social a nivel de pre y postgrado y Trabajo social crítico. En ESWRA las líneas son: Política social, Estado y las fuerzas económicas, Educación en Trabajo social y Trabajo multiprofesional. Finalmente, en el caso de la Red de Investigadores de Chile las líneas declaradas son los Debates meta disciplinarios y enfoques teóricos en trabajo social, Educación y formación en trabajo social a nivel de pre y postgrado y Política públicas y políticas sociales. En este sentido son ejes investigativos transversales los temas de formación en trabajo social a nivel de pre y postgrado, políticas sociales e intervención social.

La única red que muestra elementos explícitos de incidencia política es ABEPSS a nivel nacional y regional, con alcances y debates que involucran a sujetos sociales y colectivos en contextos de desigualdades, transformaciones contemporáneas y reconocimiento de derechos. En la Red de Investigadores en Trabajo Social de Chile, se infiere una orientación en este sentido, que aún no se plasma en las acciones desplegadas a la fecha.

Finalmente, los alcances de las asociaciones analizadas son diferentes en términos espaciales. SSWR se define con alcance nacional para los Estados Unidos, mientras que ESRWA hace lo mismo a nivel europeo. ABEPSS y Red de Investigadores tienen una definición de alcance nacional, que en el caso de la red brasilera alcanza una extensión amplia de centros y redes regionales, mientras que en la red de Chile su alcance es mucho más singular y acotado a sus socias/os.

A partir de lo anterior se observa que la tendencia a conformar redes de investigación sigue distintas lógicas que son comunes no sólo para la disciplina del trabajo social sino a las formas de concebir la producción y reproducción de conocimiento de otras disciplinas. Por un lado, encontramos aquellas concepciones que entienden la generación el conocimiento como una tarea de construcción colectiva y no solipcista, de una matriz más bien colaborativa que concibe el conocimiento como parte de un modelo de producción comp artida (Latour, 2008 [2007]), donde participan legos y expertos. 
Por otro lado, se ubica una tendencia más jerárquica, asentada en un o unos pocos investigadores, que encuentra similitudes en las redes de intelectuales y con los conceptos de consagración y capital cultural trabajado por Bourdieu (2012[1984]) y Latour (2008[2007]. Estas redes se diferencian de las redes sociales o de colegaje, como las propuestas por Sierra (1998), principalmente porque se ubican en los espacios académicos, mientras que las redes más colaborativas operarían más frecuentemente en espacios o ámbitos de intervención profesional (Chadi, 2000; Mascareño, 2010). En lógicas de reproducción social, la conformación de redes tenderá a seguir una estructura más bien jerárquica que diferencia entre investigaciones más consagrados de aquellos que se inician. En ese sentido la red tiende a reproducir las lógicas del trabajo académicos, las membresías y las formas de construcción de conocimiento basado en el trabajo de expertos, que se distancia de los modelos de producción más compartidos (Callon, 1999).

De este modelo se desprenden nociones de redes con reconocida experiencia y competencia en un dominio en particular, como ocurre en este caso con SSWR y ABEPSS en la generación de conocimiento disciplinar, lo que conlleva un cierto reclamo de autoridad que se asienta en las creencias compartidas, juicios profesionales, nociones de validez y una cierta agenda política común (Haas, 1980 y 1990). Como puntos a destacar se observa que en SSWR se centra particularmente en temáticas focalizadas, actualmente sobre juventud y salud, comunidad y paternidad/tutoría. De esta manera, se refieren a casos particulares y no se realizan alcances políticos al respecto más allá de la necesidad de políticas en algunas temáticas. Una dirección diferente es la que asume ESWRA que se ha centrado en la promoción de educación en trabajo social, tomando la educación como una herramienta para la justicia social, sin embargo, se limita la discusión política que va más allá de la profesión.

A nivel Latinoamericano, la ABEPSS explicita la relevancia de la capacitación en trabajo social, situando la intervención profesional en un contexto nacional de mercantilización que conlleva precarización. Articula elementos macro y dimensiones micro con el objetivo de potenciar el conocimiento dentro de la profesión. La red de investigadores chilena, a diferencia de las anteriores, presenta sus líneas de investigación, alcances y objetivos de una manera más explícita que las otras redes y posee un potencial de incidencia en asuntos de interés público cuyos resultados o impactos precisan ser analizados con mayor detención en futuros estudios.

\section{Conclusiones}

Los hallazgos aquí presentados sugieren que la manera en que se produce y se difunde el conocimiento incentivado por las políticas de fomento a la investigación a nivel nacional e internacional sigue estando apegada a las lógicas del capitalismo cognitivo especialmente en lo que refiere a las revistas académicas indexadas en los cuatro 
catálogos estudiados. Adicionalmente, es posible observar que la forma en que se produce conocimiento en trabajo social -tanto en lo que respecta a las revistas como a las redes de investigación- sigue siendo "insular" o geopolíticamente concentrada, con asimetrías observables en términos de fundamentación conceptual y metodológica y configuración de los equipos, y con alcances de incidencia pública diferenciados. Por una parte, las revistas tienden a reproducir lógicas endogenistas; mientras que las redes de investigación que funcionan con lógicas descentradas o heterárquicas se orientan a abrir espacios de relaciones y a ejercer influencia en el debate público y en la formación de futuros investigadoras/es desde la disciplina. Estos hallazgos permiten problematizar las lógicas de las políticas de fomento de la investigación y difusión del conocimiento -fondos públicos, incentivos, políticas de acceso- y proyectar los desafíos de trabajo social en términos de su posicionamiento y contribuciones al debate en esta materia.

Los desafíos disciplinares en lo que respecta a las revistas académicas refieren principalmente a la articulación de la discusión conceptual, el alcance político, la comprensión cosmopolita -decolonial- de la producción intelectual, que aspire a publicar trabajos bajo altos estándares metodológicos y éticos, y en revistas que garanticen el acceso abierto y gratuito. En este sentido, la formación de redes de investigación horizontales, asentadas en lógicas de intercambio y colaboración, emergen como un contrapunto frente a la impronta dominante del capitalismo cognitivo. Ciertamente las redes de investigación también presentan puntos críticos: no todos los actores que participan en la red tienen el mismo peso e influencia. Este aspecto se constituye en un elemento clave de análisis, que reconoce desde el inicio la existencia de desequilibrios a favor de algunos investigadores/as, que inciden en su legitimidad o en la valoración de conocimiento producido desde determinados cánones, enfoques o lógicas.

Lo anterior tiene implicancias para la discusión disciplinar y la forma de concebir la producción de conocimiento en tanto da cuenta de un imaginario que se comprende y se construye de manera relacional. Esto significa comprender la producción de conocimientos en el trabajo social como una red de relaciones sociales (Bourdieu, 2013 [1989] y 2012 [1984]) donde se interrelacionan y conectan actores unipersonales, equipos de investigación, instituciones y recursos, y desde las cuales se pueden ejercer resistencias frente a las lógicas del capitalismo cognitivo (Bourdieu, 1998). Aproximarse a la producción de conocimientos de este modo implica reconocer las interacciones que se dan entre generaciones y al interior de cada generación, pero también las individualidades de cada trayectoria, las tensiones y disputas en torno a la autorías y membresías, los límites geopolíticos desde donde el conocimiento ha sido construido y las conexiones que se establecen con otras/os investigadores al interior de la disciplina y con otras disciplinas relacionadas. 
Estamos seguras de que los debates en torno a producción de conocimiento en general y en trabajo social en particular, están experimentando cambios y transformaciones. Así lo expresa César Hidalgo en la entrevista hecha por Cabezas (2020), al reconocer que la ciencia es global y que por lo mismo "el concepto país no tiene mucho sentido". El conocimiento geopolíticamente referenciado reproduce lógicas endogenistas y encapsula y captura a las/os investigadores en sus redes de producción y legitimación. Identificar sus tendencias, orientaciones y dinámicas nos permite comprender mejor el carácter político y situado del conocimiento, su posibilidad de incidencia y las resistencias que pueden surgir ante estas lógicas dominantes.

Evidenciar y reconocer estos procesos nos alienta a abordar el centenario del trabajo socia en Chile y América Latina con desafíos actualizados, que inviten a resistir en las posiciones de un trabajo colaborativo frente a los principios del capitalismo cognitivo, construyendo conocimientos de manera horizontal y con perspectiva intergeneracional. Tenemos esperanzas en torno a lo que está por venir en estos sentidos.

\section{Referencias bibliográficas}

Alperín, J.P y Fischman, G. (Eds.). (2015). Hecho en Latinoamérica: acceso abierto, revistas académicas e innovaciones regionales. CLACSO.

Álvarez-Uría, F. y Parra, P. (2014). The bitter Cry: materiales para una genealogía de la identidad profesional de las pioneras del Trabajo Social en Inglaterra y Estados Unidos. Cuadernos de Trabajo Social, 27, 95-194.

Blondeau, O., Dyer Whiteford, N., Vercellone, C., Kyrou, A., Corsani, A., Rullani, E., Moulier Boutang, Y. y Lazzarato, M. (2004). Capitalismo cognitivo. Propiedad intelectual y creación colectiva. Traficantes de Sueños.

Bourdieu, P. (1998). Acts of Resistance: Against the Tyranny of the Market. Polity Press.

Bourdieu, P. (2012 [1984]). Homo academicus. Siglo XXI

Bourdieu, P. (2013 [1989]). La nobleza de Estado. Siglo XXI.

Cabezas, E. (2020, 14 de noviembre). Me encantaría que ser global fuera más accesible. Entrevista a César Hidalgo. Revista Sábado de El Mercurio.

Callon, M. (1999) El Rol de los Ciudadanos en la Producción y Divulgación de Conocimiento Científico. Ciencia, Tecnología y Sociedad, $4, \quad 81-94$. http://sts.sagepub.com/content/4/1/81.full.pdf+html [6. febrero 2015]

Chadi, M. (2000) Redes Sociales en el Trabajo Social. Espacio.

D’Amico, M. (2016). Presentación. En F. Sierra (Coord.), Capitalismo cognitivo y economía social del conocimiento. La lucha por el código (pp. 432 - 436). Ciespal. 
Deegan, M. J. (1997). The Chicago Men and the Sociology of Women. En K. Plummer (Edit.), The Chicago School. Critical Assessments, Vol. I. (pp 198 y 214). Routledge.

Fardella, C., Jiménez, F. y Sisto, V. (2017). La transformación de la universidad y los dispositivos de cuantificación. Estudos de Psicología , 34(3), 435-448.

Fardella, C., Corvalán, A. y Zavala, R. (2019). El académico cuantificado. La gestión performativa a través de los instrumentos de medición en la ciencia. Psicología, Conocimiento y Sociedad 9(2), 77-103.

Figueroa, Y., Chamblas, I. y Rubilar, G. (2018). La generación de conocimiento en Trabajo Social: percepción de graduadas y graduados de dos programas de Magíster en Trabajo Social de Chile, Cuadernos de trabajo social, 31(2), 407-416.

Haas, E. B. (1980). Why Collaborate? Issue-Linkage and International Regimes. World Politics, 32, 357-405.

Haas, E. B. (1990). When Knowledge is Power. University of California Press.

Hott, E. (1930). Medios para mantener el ideal en el servicio social. Revista Servicio Social 4(4), 25-46.

Lander, E. (1999.) ¿Conocimiento para qué? ¿Conocimiento para quién? Reflexiones sobre la universidad y la geopolítica de los saberes hegemónicos. Estudios Latinoamericanos, 7(12), 25- 46. http://dx.doi.org/10.22201/cela.24484946e.1999.12-13.52369

Latour, B. (2008 [2007]). Reensamblar lo social: una introducción a la teoría del actor-red. Manantial.

Lee, H. \& Lee, K. (2013). Publish (in international indexed journals) or perish: Neoliberal ideology in a Korean university. Language Policy, 12, 215-230.

Lillis, T. \& Curry, M.J. (2010). Academic Writing in a Global Context. The politics and practices of publishing in English. Routledge.

Mascareño, A. (2010). Coordinación social mediante políticas públicas: el caso chileno. Revista de la Cepal, 101, 111-126.

Matus, T., Aylwin, N. y Forttes, A. (2004). La reinvención de la memoria. Indagación sobre el proceso de profesionalización del trabajo social chileno 1925-1965. P. Universidad Católica de Chile. 


\section{ARTÍCULO}

Miranda, M. (2003). El compromiso con la ciencia. Conocimiento y técnica en el Trabajo Social. Revista Internacional de Ciencias Sociales y Humanidades SOCIOTAM, XVII(2), 9- 28.

Morera, N. (2010). El vínculo entre las ciencias sociales y el Trabajo Social: algunos elementos para la discusión. Revista Reflexiones, 89(1), 235-241.

Muñoz, G. (2018). Razón neoliberal e investigación: resistencias desde el trabajo social. Cuadernos de Trabajo Social, 17, 32-54.

Muñoz, G. \& Rubilar, G. (2020). Social Work Research in Chile: Tensions and Challenges under the 'Knowledge Economy' and Managerialist Research Agendas. The British Journal of Social Work, 1-18. doi: 10.1093/bjsw/bcaa132

Murray, G. \& Peetz, D. (2020). Has Exploitation Transformed? A Critical Analysis of the Theory of Cognitive Capitalism. Perspectives on Global Development and Technology, 19(1-2), 17-31.

Ossa, C. (2016). El ego explotado. Capitalismo cognitivo y precarización de la Creatividad.

Ediciones Departamento de Artes Visuales Universidad de Chile.

Reininger, T. (2018). El movimiento de asentamiento: el valioso legado de Jane Addams para un trabajo social radical. En B. Castro y M. Flotts (Eds.). Imaginarios de transformación. El trabajo social revisitado (pp. 73-96). RIL.

Roche, S. y Flynn, K. (2018). Geographical inequity in social work research: A snapshot of research publications from the global South. International Social Work, 63(3), 306-322.

Serrano, J. y Prats, J. (2005). Repertorios abiertos: el libre acceso a los contenidos. Revista de Universidad y Sociedad del Conocimiento, 2(2). https://www.redalyc.org/pdf/780/78020109.pdf

Sierra, F. y Alberich, J. (Eds.) (2019). Epistemología de la comunicación y cultura digital. Retos emergentes. Universidad de Granada.

Smith, R. (2006). The power of the unrelenting impact factor-Is it a force for good or harm? International Journal of Epidemiology, 35, 1129-1130.

Tarragó, N., Santiago, L. y Macedo dos Santos, R. (2018). La producción científica latinoamericana desde una mirada poscolonial. https://latinoamericanarevistas.org/?p=138 
Taylor, B. J. y Sharland, E. (2015). The Creation of the European Social Work Research Association. Research on Social Work Practice, 25(5), 623-627. doi:10.1177/1049731514558686

Torres, C. A. (2014). Las universidades públicas y el sentido común neoliberal: siete tesis iconoclastas. Revista Latinoamericana de Políticas y Administración de la Educación, 1(1), 18-31.

Travi, B. (2011). Conceptos e ideas clave en la obra de Mary Ellen Richmond y la vigencia actual de su pensamiento. Cuadernos de Trabajo Social, 24, 57-67.

Vercellone, C. (2013). From the mass-worker to cognitive labour: Historical and theoretical considerations. En M. Linder, y K. H. Roth (eds.), Beyond Marx: Theorising the Global Labour Relations of the Twenty-First Century (pp. 417-445). Koninklijke Brill.

Zuchowski, I., Miles, D., Gair, S. y Tsey, K. (2019). Social work research with industry: A systematic literature review of engagement and impact. The British Journal of Social Work, 49(8), 2299-324.

\section{Agradecimientos}

Agradecimientos a ANID/CONICYT/ FONDECYT 1190257, Proyecto Estudio Longitudinal de trayectorias y transiciones investigativas de trabajadores sociales chilenos (2019-2023) y a ANID/CONICYT/FONDECYT 1201685, Proyecto Resistencias profesionales en la primera línea de implementación de programas sociales (2020-2023).

\section{Biografía de las autoras}

Gianinna Muñoz - Arce es Doctora en Trabajo Social, Académica del Departamento de Trabajo Social y Coordinadora del Núcleo de Estudios Interdisciplinarios en Trabajo Social de la Universidad de Chile. Líneas de investigación: debates disciplinarios en trabajo social, teorías críticas e intervención social, implementación de programas sociales. Correo electrónico: gianinna.munoz@uchile.cl. ORCID ID: https://orcid.org/0000-0003-4582-0507

Gabriela Rubilar - Donoso es Doctora en Metodología de Investigación por la Universidad Complutense de Madrid y Doctora en Ciencias Humanas y Sociales por la Universidad Pontificia Comillas de Madrid, España. Es académica y directora del Departamento de Trabajo Social de la Universidad de Chile. Integrante del Núcleo de 
Estudios Interdisciplinarios en Trabajo Social. Líneas de investigación: Políticas públicas y programas sociales, pobreza y exclusión, investigación en Trabajo Social. Correo electrónico: grubilar@uchile.cl. ORCID ID: http://orcid.org/0000-0002-4635-9380

Teresa Matus - Sepúlveda es Doctora en Trabajo Social por la Universidad Federal de Río de Janeiro y Doctora en Sociología por la Universidad Candido Mendes IUPERJ. Académica y subdirectora del Departamento de Trabajo Social de la Universidad de Chile. Integrante del Núcleo de Estudios Interdisciplinarios en Trabajo Social. Líneas de investigación: teorías en trabajo social, epistemología de las ciencias sociales e innovaciones en políticas públicas.Correo electrónico: teresamatus@uchile.cl. ORCID ID: https://orcid.org/0000-0002-2974-9678

Paula Parada - Ballesteros es Licenciada en Trabajo Social y cursa su último año de formación profesional en la Universidad de Chile. Es integrante del Núcleo de Estudios Interdisciplinarios en Trabajo Social de la Universidad de Chile, Diplomada en Sexualidad Transdisciplinaria Etsex de la Universidad de Chile. Participa del Colectivo Mujeres Autoconvocadas de Macul. Sus intereses de investigación son sexualidades, salud, resistencias profesionales, innovación y políticas sociales. Correo electrónico: paula.parada@ug.uchile.cl. ORCID ID: https://orcid.org/0000-0002-4150-725X 